\title{
Gram-positive pathogenic bacteria induce a common early response in human monocytes
}

\author{
Svetlin Tchatalbachev ${ }^{1 *}$, Rohit Ghai ${ }^{1,2}$, Hamid Hossain $^{1}$, Trinad Chakraborty ${ }^{1}$
}

\begin{abstract}
Background: We infected freshly isolated human peripheral monocytes with live bacteria of three clinically important gram-positive bacterial species, Staphylococcus aureus, Streptococcus pneumoniae and Listeria monocytogenes and studied the ensuing early transcriptional response using expression microarrays. Thus the observed response was unbiased by signals originating from other helper and effector cells of the host and was not limited to induction by solitary bacterial constituents.

Results: Activation of monocytes was demonstrated by the upregulation of chemokine rather than interleukin genes except for the prominent expression of interleukin 23, marking it as the early lead cytokine. This activation was accompanied by cytoskeleton rearrangement signals and a general anti-oxidative stress and anti-apoptotic reaction. Remarkably, the expression profiles also provide evidence that monocytes participate in the regulation of angiogenesis and endothelial function in response to these pathogens.

Conclusion: Regardless of the invasion properties and survival mechanisms of the pathogens used, we found that the early response comprised of a consistent and common response. The common response was hallmarked by the upregulation of interleukin 23, a rather unexpected finding regarding Listeria infection, as this cytokine has been linked primarily to the control of extracellular bacterial dissemination.
\end{abstract}

\section{Background}

The percentage of patients with severe infections caused by gram-positive bacteria has increased in recent years, accounting for almost half of the incidents of septicemia and severe systemic infections [1-5]. A number of recent publications have investigated the transcriptional response to killed or inactive gram-positive pathogens, or the contribution of gram-positive cell wall constituents such as peptidoglycan (PepG), lipopeptide (LP) and lipoteichoic acid (LTA) to the triggering of specific host defense responses [6-10]. Though such studies are crucial for identifying stimulus specific effects, they are unable to account for the immunomodulatory effects of live bacteria, which frequently employ multiple survival strategies in parallel. Viable pathogenic bacteria secrete active components in the intercellular space and in the invaded cells in order to modulate the cellular response. In order to track the early events of gram-positive

\footnotetext{
* Correspondence: Svetlin.Tchatalbachev@mikrobio.med.uni-giessen.de ${ }^{1}$ Institute of Medical Microbiology, Justus Liebig University, Frankfurter Str. 107, 35392 Giessen, Germany

Full list of author information is available at the end of the article
}

induced immune activation, we examined the total transcriptional response of isolated peripheral human CD14+/CD11b+ monocytes, infected with the viable bacterial pathogens: Listeria monocytogenes, Staphylococcus aureus and Streptococcus pneumoniae (hereafter referred to as LM, SA and SP respectively). All three pathogens belong to the group of low GC content bacteria. SP and SA are leading pathogens in cases of gram-positive sepsis and LM is a cause of meningitis in immunocompromised patients and also sepsis in newborns.

We designed and established a protocol enabling the detection of pathological changes early in the onset of infections with gram positive pathogens, before usual clinical parameters are upregulated, in an easily accessible cellular sample material. For these purposes, we focused our experimental analysis of naïve monocytes, which are easier to work with in ex vivo conditions than granulocytes, even though they are represented in much lower numbers in vivo than the latter. Peripheral monocytes also are among the first members of the host immune system to encounter pathogens after injury and epithelial penetration. We limited the infection to a 
short interval of 1 hour in the attempt to mimic the in vivo early reaction of the cells after first encountering the pathogen but before the onset of clinically manifested inflammation. Using microarray analysis, we were able to detect the transcriptional upregulation or repression of a robust minimal set of genes in infected cells compared to untreated controls in the short interval of one hour. Despite donor specific gene variations and despite the different invasion strategies of the bacteria studied, we identified a common program of gene expression induced by all three bacterial pathogens. This program is characterized by the upregulation of a key cytokine - interleukin 23 (IL23).

\section{Results}

\section{Global response pattern of peripheral monocytes to infection}

To assess the global response we performed clustering of the correlation coefficients of the entire gene expression matrix comprising the unchallenged and the infected monocytes with all three pathogens (Figure 1). This revealed an interesting pattern. As can be seen from the figure, there are three main clusters. Cluster A comprising the controls, Cluster B comprising infection with L. monocytogenes (LM) and S. aureus (SA), and Cluster C comprising infection with $S$. pneumoniae (SP). We compared each pathogen to the control group to identify the differentially expressed genes (Figure 2). LM caused the induction of transcription of 205 and repression of 233 genes (Figure 2A; Additional files 1, 2, Tables S1, S2). The transcription of 192 genes was upregulated and 171 genes were downregulated upon infection with SA (Figure 2A; Additional files 3, 4, Tables S3, S4). For SP these numbers were smaller, with 102 and 38 genes upregulated respectively downregulated $1 \mathrm{~h}$ upon infection (Figure 2A; Additional files 5, 6, Tables $\mathrm{S} 5$, S6). Induction of target gene expression for the common upregulated genes was consistently higher for LM and SA than SP. All differentially expressed genes by pathogen with fold changes are available as additional files (Additional files 1, 2, 3, 4, 5, 6, Tables S1-S6).

\section{Common and pathogen specific responses of peripheral monocytes}

All pathogens induced a common set of 66 upregulated and 32 downregulated genes (Tables 1, 2, Figure 2B). Consistent with common core responses against pathogenic stimuli [11], we observed genes involved in proinflammation, chemotaxis, suppression of immune response and adhesion molecules. LM induced the largest number of pathogen-specific transcription changes, especially downregulating 95 genes (Figure 2B; Additional files 7, 8, Tables S7, S8), compared with 34 by SA (Figure 2B; Additional files 9, 10, Tables S9, S10). Only two genes (out of a total of 38 downregulated) were individually downregulated by SP and 20 genes were upregulated only by infection with SP (Figure 2B; Additional files 11, 12, Tables S11, S12). All of the common regulated genes sorted by Gene Ontology (GO) are available as additional file (Additional file 13, Excel work sheet S1).

Surprisingly, the major inflammatory cytokines IL1 and TNF were absent from the list of commonly expressed genes, and highest expression levels in this gene set were detected for the interleukin 23A (IL23, p19) mRNA followed by CD248 (CD164 sialomucinlike 1). In our experiment the IL23A levels were 48 -, 30- and 6-fold elevated after infection with LM, SA and $\mathrm{SP}$, respectively. We observed induction of chemokines and cytokines of the CCL and CXCL families with CCL8 and CCL14 commonly induced by all thee pathogens. Genes responsible for the rearrangement of the cytoskeleton and adhesion e.g. talin2 (TLN2), claudin 1 (CLD1) and tubulin complex member protein 6 (TUBGCP6) were also upregulated (Table 1).

\section{Variation in the control group}

The availability of non-infected controls provided us with an opportunity to assess which genes are most differently expressed among the control group before any challenge with the bacteria as well. The top most variable 100 genes were analyzed using the over-presentation analysis feature in the DAVID database (see Methods) using the entire list of accessions from the microarray chip as a background. We found that the gene categories that were highly over-represented in this set of genes (e.g. defensins B1, complement factor B, adenosine $\mathrm{a} 2 \mathrm{~b}$ receptor, inhibin) were related to the immune response. This suggests that even without bacterial challenge, gene expression of the immune response genes is highly variable and this may also reflect the different physiological state of the donors. At the other end of the spectrum, the genes with the lowest variation in gene expression were found to comprise genes responsible for cellular adhesion (integrin alpha1, tenascin, muskelin 1 etc.), cytoskeleton organization (desmin, kinesin etc.) and oxidative phosphorylation (ATP synthase subunits and cytochrome c oxidase) suggesting that maintenance of cell-cell interactions, cellular shape and energy generation are important functions that are discharged in a uniform manner across donors. The list of these most variable and the least variable genes across all donors is available as additional file (Additional file 14, Excel work sheet S2).

\section{Validation of microarray data by quantitative RT-PCR (qRT-PCR)}

In order to verify our microarray data we performed qRT-PCR with 14 target genes. IL23A (Interleukin 23 


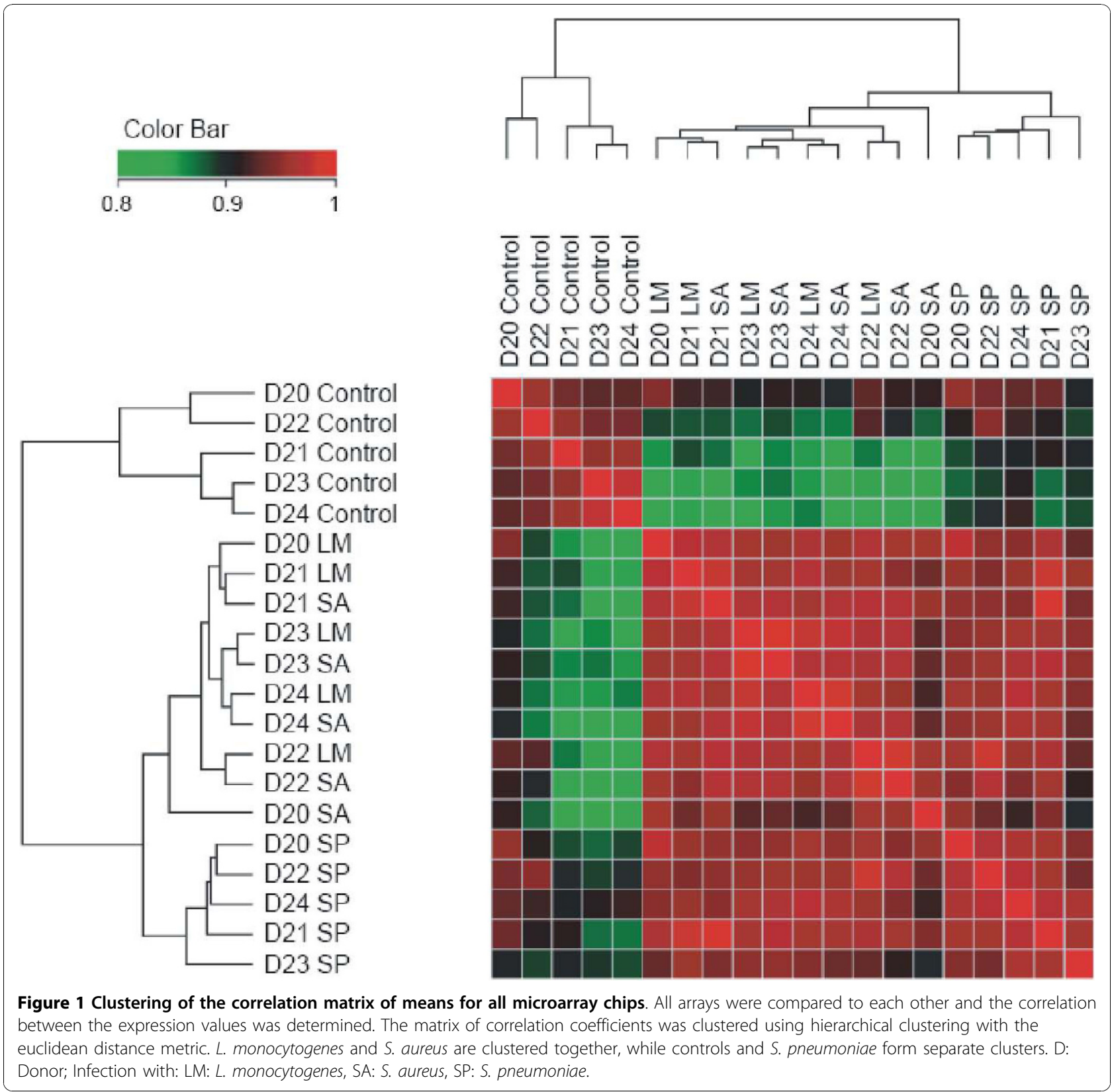

alpha subunit, p19), JUN (Jun oncogene), NALP2 (NLR family, pyrin domain containing 2), FADD (Fas (TNFRSF6)-associated via death domain), SOCS3 (Suppressor of cytokine signaling 3), SOCS5 (Suppressor of cytokine signaling 5), TLR1 (Toll like receptor 1), SAA (Serum amyloid A2), IL21R (Interleukin 21 receptor), DEFB1 (Defensin beta 1), IL15RA (Interleukin 15 receptor, alpha), PSMB9 (Proteasome subunit beta type 9), IL10 (Interleukin 10) and INHBA (Inhibin beta A). The relative fold change of target genes was normalized by the relative expression of a pool of 4 reference genes: B2M (Beta 2 microglobulin), G6PD (Glucose 6 phosphate dehydrogenase), PGK1 (Phosphoglycerate kinase 1) and SDHA (Succinate dehydrogenase alpha subunit). Normalized fold change for a target gene versus every reference gene was calculated and a mean fold change of these four was the final value. This normalized mean fold change was plotted against the microarray expression fold change for the same target gene and the linear regression showed a correlations coefficient $\mathrm{R}^{2}=0.914$ (Additional file 15, Figure S1).

\section{IFN $\gamma$, IL12A and IL23B expression}

Since the CodeLink human UniSet I array does not contain a probe for interferon gamma (IFN $\gamma$ ), we 


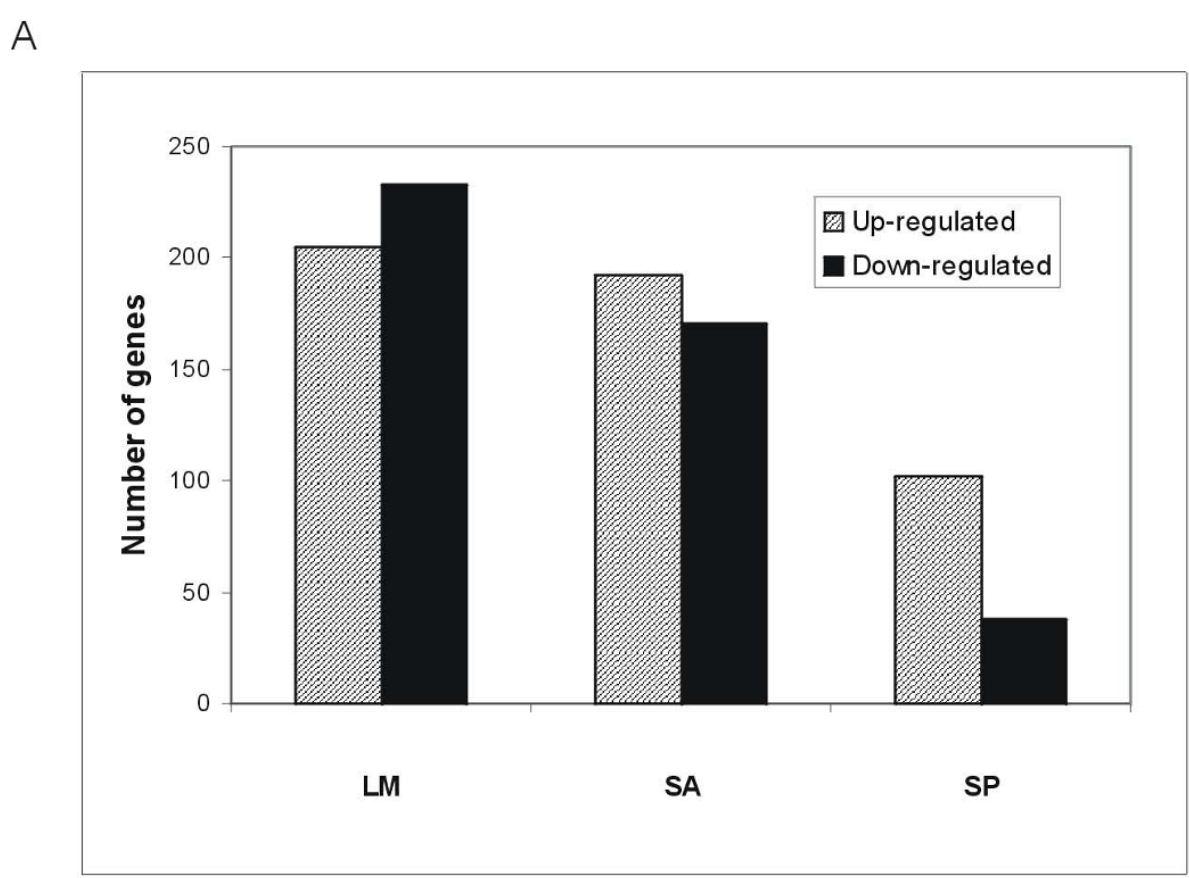

B

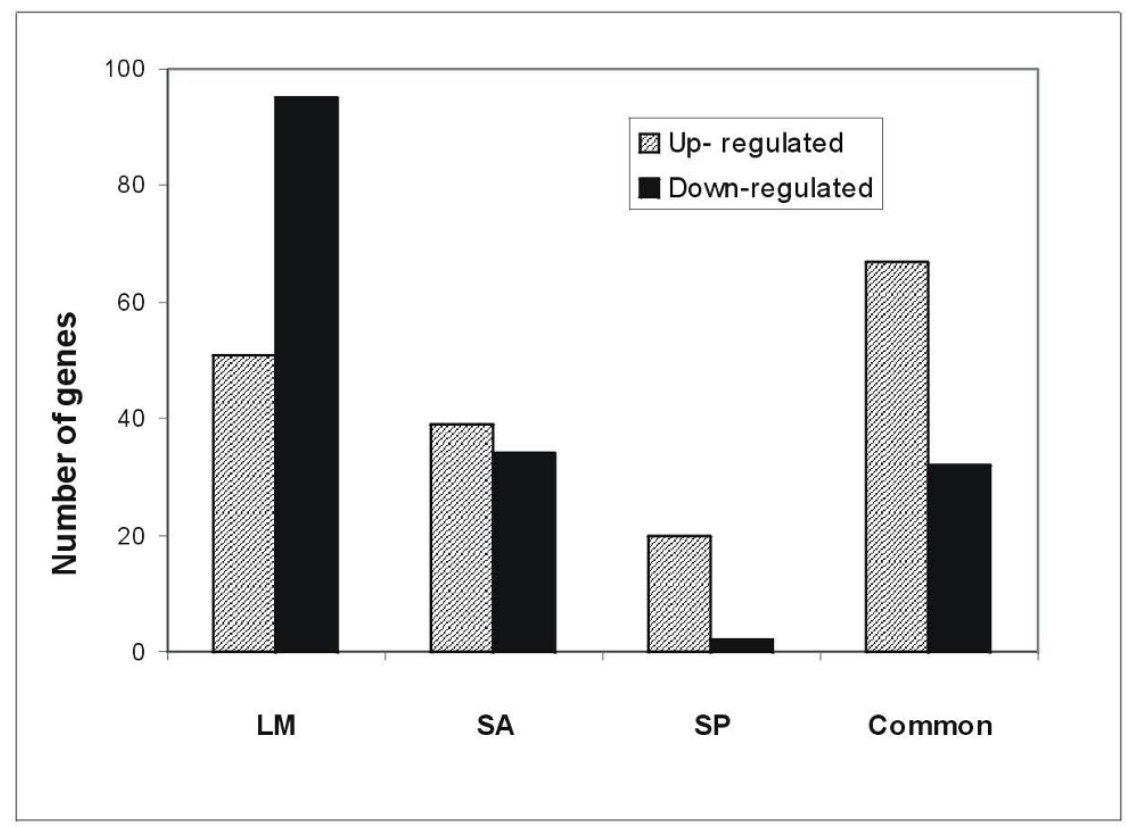

Figure 2 Differentially expressed genes induced by each pathogen. (A) Total upregulated and downregulated genes by each pathogen are represented as fold change values compared to the expression of the non-infected sample. (B) Comparison of specific and common induction of differentially expressed genes by each pathogen alone and by all three. Listeria monocytogenes induces the strongest common and specific gene regulation of all three pathogens fallowed by S. aureus and S. pneumoniae. LM: L. monocytogenes EGDe, SA: S. aureus, SP: S. pneumoniae.

additionally performed real time RT-PCR tests with IFN $\gamma$ specific primers and found the mRNA to be 9.5 fold upregulated by LM, 6.2 fold induced by SA and 1.8 fold induced by SP (Figure 3; Additional file 16, Table
S13). We also evaluated the relative expression of IL12A (p35) and IL23B (IL12B) mRNAs. IL12 and IL23 are heterodimeric cytokines, which share the same beta subunit, a protein of $40 \mathrm{KDa}$ (IL12B/IL23B-p40). The 
Table 1 List of commonly upregulated genes for all pathogens.

\begin{tabular}{|c|c|c|c|c|c|c|}
\hline & & & & Folc & d Chan & \\
\hline No. & $\begin{array}{l}\text { Gene } \\
\text { IDs }\end{array}$ & $\begin{array}{l}\text { Gene } \\
\text { Symbol }\end{array}$ & Gene name & LM & SA & SP \\
\hline 1 & 51561 & IL23A & Interleukin 23, alpha subunit p19'"' & 48,15 & 30,02 & 6,70 \\
\hline 2 & 57124 & CD164L1 & CD248 antigen, endosialin"'"' & 20,36 & 22,03 & 8,23 \\
\hline 3 & 3426 & IF & I factor (complement) & 17,07 & 13,08 & 6,88 \\
\hline 4 & 10411 & RAPGEF3 & Rap guanine nucleotide exchange factor (GEF) 3 & 13,85 & 11,81 & 5,60 \\
\hline 5 & 1960 & EGR3 & Early growth response 3 & 12,16 & 8,98 & 2,65 \\
\hline 6 & 3725 & JUN & V-jun sarcoma virus 17 oncogene homolog (avian) & 10,80 & 12,09 & 6,35 \\
\hline 7 & 9389 & SLC22A14 & Solute carrier family 22 (organic cation transporter), member 14"'"' & 10,41 & 9,29 & 5,40 \\
\hline 8 & 6648 & SOD2 & Superoxide dismutase 2, mitochondrial'"'" & 10,24 & 8,61 & 4,23 \\
\hline 9 & 3290 & HSD11B1 & Hydroxysteroid (11-beta) dehydrogenase 1 & 10,04 & 20,04 & 5,73 \\
\hline 10 & 122616 & C14orf79 & Chromosome 14 open reading frame 79 & 9,27 & 8,15 & 4,45 \\
\hline 11 & 4790 & NFKB1 & Nuclear factor of kappa light polypeptide gene enhancer in B-cells 1 (p105) & 8,03 & 8,17 & 3,06 \\
\hline 12 & 131566 & DCBLD2 & Discoidin, CUB and LCCL domain containing 2"'"' & 7,94 & 5,46 & 4,59 \\
\hline 13 & 1906 & EDN1 & Endothelin 1 & 7,76 & 10,71 & 3,40 \\
\hline 14 & 9025 & RNF8 & Ring finger protein 8 & 7,38 & 6,01 & 2,98 \\
\hline 15 & 51458 & RHCG & Rhesus blood group, C glycoprotein"'"' & 6,90 & 8,94 & 2,55 \\
\hline 16 & 1735 & $\mathrm{DlO} 3$ & Deiodinase, iodothyronine, type III"'"' & 6,83 & 6,20 & 3,70 \\
\hline 17 & 9021 & SOCS3 & Suppressor of cytokine signaling 3 & 6,82 & 9,90 & 3,10 \\
\hline 18 & 83740 & H2AFB3 & H2A histone family, member B3'"' & 6,69 & 8,58 & 3,55 \\
\hline 19 & 9028 & RHBDL1 & Rhomboid, veinlet-like 1 (Drosophila)'"'" & 6,18 & 5,07 & 2,28 \\
\hline 20 & 8659 & $\mathrm{ALDH} 4 \mathrm{~A} 1$ & Aldehyde dehydrogenase 4 family, member $A 11^{\prime \prime \prime \prime}$ & 6,13 & 3,74 & 3,02 \\
\hline 21 & 8740 & TNFSF14 & Tumor necrosis factor (ligand) superfamily, member 14'"' & 5,74 & 3,83 & 1,60 \\
\hline 22 & 51025 & Magmas & $\begin{array}{l}\text { Mitochondria-associated protein involved in granulocyte-macrophage colony-stimulating factor } \\
\text { signal transduction }\end{array}$ & 5,52 & 5,54 & 3,11 \\
\hline 23 & 10120 & ACTR1B & ARP1 actin-related protein 1 homolog B, centractin beta (yeast)'"'" & 5,17 & 4,63 & 2,70 \\
\hline 24 & 2615 & GARP & Leucine rich repeat containing 32 & 4,96 & 3,18 & 3,03 \\
\hline 25 & 55655 & NALP2 & NACHT, leucine rich repeat and PYD containing 2'"'" & 4,72 & 4,59 & 2,83 \\
\hline 26 & 6004 & RGS16 & Regulator of G-protein signalling 16 & 4,66 & 2,42 & 2,22 \\
\hline 27 & 6935 & TCF8 & Transcription factor 8 (represses interleukin 2 expression) & 4,66 & 2,81 & 7,11 \\
\hline 28 & 3280 & HES1 & Hairy and enhancer of split 1, (Drosophila)'"'" & 4,65 & 4,37 & 2,02 \\
\hline 29 & 983 & CDC2 & Cell division cycle 2, G1 to $\mathrm{S}$ and $\mathrm{G} 2$ to $\mathrm{M}^{\prime \prime \prime \prime \prime}$ & 4,64 & 4,21 & 2,31 \\
\hline 30 & 9076 & CLDN1 & Claudin 1 & 4,60 & 4,85 & 2,59 \\
\hline 31 & 10307 & APBB3 & Amyloid beta (A4) precursor protein-binding, family B, member $3^{\prime \prime \prime \prime \prime}$ & 4,53 & 3,47 & 2,90 \\
\hline 32 & 283131 & TncRNA & Trophoblast-derived noncoding RNA & 4,29 & 5,03 & 2,53 \\
\hline 33 & 114112 & TXNRD3 & Thioredoxin reductase 3 & 4,15 & 3,55 & 2,01 \\
\hline 34 & 6355 & CCL8 & Chemokine (C-C motif) ligand 8 & 4,06 & 3,54 & 2,54 \\
\hline 35 & 1164 & CKS2 & CDC28 protein kinase regulatory subunit 2 & 3,95 & 3,12 & 2,11 \\
\hline 36 & 5142 & PDE4B & Phosphodiesterase 4B, CAMP-specific (phosphodiesterase E4 dunce homolog, Drosophila)"'"' & 3,93 & 3,93 & 2,35 \\
\hline 37 & 6358 & CCL14 & Chemokine (C-C motif) ligand 14 & 3,80 & 4,33 & 3,76 \\
\hline 38 & 788 & SLC25A20 & Solute carrier family 25 (carnitine/acylcarnitine translocase), member $20^{\prime \prime \prime \prime}$ & 3,72 & 3,69 & 2,55 \\
\hline 39 & 3697 & $\mathrm{ITIH1}$ & Inter-alpha (globulin) inhibitor H1 & 3,71 & 3,12 & 2,05 \\
\hline 40 & 2322 & FLT3 & Fms-related tyrosine kinase 3 & 3,66 & 4,59 & 2,20 \\
\hline 41 & 6489 & SIAT8A & ST8 alpha-N-acetyl-neuraminide alpha-2,8-sialyltransferase $1^{\prime \prime \prime \prime}$ & 3,47 & 3,23 & 2,67 \\
\hline 42 & 23529 & $\mathrm{CLC}$ & Cardiotrophin-like cytokine factor 1 & 3,33 & 7,64 & 2,12 \\
\hline 43 & 55647 & RAB20 & RAB20, member RAS oncogene family"'"' & 3,31 & 3,86 & 3,95 \\
\hline 44 & 54847 & SIDT1 & SID1 transmembrane family, member $1^{\prime \prime \prime \prime}$ & 3,15 & 4,43 & 2,78 \\
\hline
\end{tabular}


Table 1: List of commonly upregulated genes for all pathogens. (Continued)

\begin{tabular}{|c|c|c|c|c|c|c|}
\hline 45 & 10514 & MYBBP1A & MYB binding protein (P160) 1a & 3,09 & 5,53 & 2,35 \\
\hline 46 & 64108 & IFRG28 & $28 \mathrm{kD}$ interferon responsive protein & 3,08 & 2,48 & 2,22 \\
\hline 47 & 9590 & AKAP12 & A kinase (PRKA) anchor protein (gravin) 12 & 2,99 & 2,72 & 2,09 \\
\hline 48 & 5894 & RAF1 & V-raf-1 murine leukemia viral oncogene homolog 1 & 2,97 & 2,75 & 2,19 \\
\hline 49 & 51365 & PLA1A & Phospholipase A1 member A & 2,95 & 4,52 & 2,45 \\
\hline 50 & 6696 & SPP1 & Secreted phosphoprotein 1 (osteopontin, bone sialoprotein I, early T-lymphocyte activation 1)'"'" & 2,93 & 5,05 & 1,70 \\
\hline 51 & 1939 & LGTN & Ligatin & 2,80 & 3,90 & 2,15 \\
\hline 52 & 57801 & HES4 & Hairy and enhancer of split 4 (Drosophila) & 2,78 & 2,72 & 2,13 \\
\hline 53 & 3202 & HOXA5 & Homeo box A5 & 2,76 & 2,07 & 2,37 \\
\hline 54 & 4216 & MAP3K4 & Mitogen-activated protein kinase kinase kinase 4 & 2,68 & 3,54 & 2,05 \\
\hline 55 & 481 & ATP1B1 & ATPase, $\mathrm{Na}+/ \mathrm{K}+$ transporting, beta 1 polypeptide'"'" & 2,65 & 2,04 & 2,27 \\
\hline 56 & 1466 & CSRP2 & Cysteine and glycine-rich protein 2 & 2,64 & 8,75 & 3,66 \\
\hline 57 & 11182 & SLC2A6 & Solute carrier family 2 (facilitated glucose transporter), member $6^{\prime \prime \prime \prime}$ & 2,48 & 2,46 & 2,00 \\
\hline 58 & 83660 & TLN2 & Talin 2 & 2,41 & 2,26 & 2,21 \\
\hline 59 & 7003 & TEAD1 & TEA domain family member 1 (SV40 transcriptional enhancer factor) & 2,40 & 1,92 & 2,11 \\
\hline 60 & 85378 & TUBGCP6 & Tubulin, gamma complex associated protein 6"'" & 2,40 & 2,72 & 2,46 \\
\hline 61 & 1846 & DUSP4 & Dual specificity phosphatase 4 & 2,38 & 2,92 & 2,60 \\
\hline 62 & 7422 & VEGF & Vascular endothelial growth factor & 2,30 & 2,27 & 2,10 \\
\hline 63 & 10560 & SLC19A2 & Solute carrier family 19 (thiamine transporter), member $2^{\prime \prime \prime \prime}$ & 2,10 & 2,45 & 2,90 \\
\hline 64 & 6617 & SNAPC1 & Small nuclear RNA activating complex, polypeptide 1, 43 kDa'"' & 2,05 & 2,65 & 2,38 \\
\hline 65 & 6515 & SLC2A3 & Solute carrier family 2 (facilitated glucose transporter), member $3^{\prime \prime \prime \prime}$ & 2,04 & 2,57 & 2,36 \\
\hline 66 & 136 & ADORA2B & Adenosine $\mathrm{A} 2 \mathrm{~b}$ receptor & 1,90 & 1,79 & 1,72 \\
\hline
\end{tabular}

FDR 10. LM: L. monocytogenes EGDe, SA: S. aureus, SP: S. pneumoniae.

combination of $\mathrm{p} 40$ with a different alpha subunit forms the physiologically active IL12 (p35p40) or IL23 (p19p40). The IL23B was not found upregulated after statistical evaluation and filtering of the primary microarray data, however IL23A (p19) mRNA was among the most strongly upregulated genes by all three pathogens and hence enhanced expression of the p40 unit was expected. The qRT-PCR data showed clearly that IL23B (IL12B) mRNA expression was increased in the monocytes of all donors. However this upregulation was highly donor-specific and varied between 2 fold and 54 fold for LM infection and reached up to more than $10^{3}$ fold change for SA (Figure 3; Additional file 16, Table S13). The expression of IL12A (p35) as demonstrated by the qRT-PCR data was regulated at a much lower level with fold change values between +2 and -2 and was also donor specific.

\section{Discussion}

Using whole-genome based microarray analysis we were able to detect the transcriptional upregulation or repression of a robust minimal set of genes in infected cells compared to untreated controls even within the short interval of one hour. Despite donor-specific gene variations and despite varying invasion strategies of the studied bacteria we identified a common program of gene expression induced by all three bacterial pathogens.

Remarkably, global comparison of the expression profiles already hinted at gross similarities by the infection among the pathogens (Figure 1, Tables 1, 2). For example, the clustering suggested that the global response of LM and SA are more similar to each other while SP infection generates a different and more subdued response pointing to similarities in the virulence of both LM and SA. One assumption may be that they generate similar responses because of their intracellular nature. However after one hour of infection we observed only a few internalized bacteria (data not shown) suggesting that secreted bacterial factors, a common feature between L. monocytogenes and S. aureus are important inducers of the response observed. LM expresses a cholesterol-dependent cytolysin (CDC) listeriolysin, that is crucial for gaining entry to the cytosol while SA encodes for several haemolysins and cytolysins e.g. the two secretory haemolysins $\alpha$ and $\beta$ [12]. SP, on the other hand, are generally encapsulated bacteria with the capsule effectively preventing ingestion of the bacteria by the monocyte. This creates a physical barrier between the bacteria and the host cell and could underlie the observations on host gene expression made here. The 
Table 2 List of commonly downregulated genes for all pathogens

\begin{tabular}{|c|c|c|c|c|c|c|}
\hline \multirow[b]{2}{*}{ No. } & \multirow[b]{2}{*}{ Gene IDs } & \multirow[b]{2}{*}{ Symbol } & \multirow[b]{2}{*}{ Gene name } & \multicolumn{3}{|c|}{ Fold Change } \\
\hline & & & & LM & SA & SP \\
\hline 1 & 55794 & DDX28 & DEAD (Asp-Glu-Ala-Asp) box polypeptide 28 & $-11,27$ & $-6,62$ & $-2,56$ \\
\hline 2 & 9529 & BAG5 & BCL2-associated athanogene 5 & $-8,73$ & $-6,03$ & $-2,56$ \\
\hline 3 & 54554 & WDR5B & WD repeat domain $5 B$ & $-8,71$ & $-6,90$ & $-2,98$ \\
\hline 4 & 80818 & ZNF436 & Zinc finger protein 436 & $-7,57$ & $-4,44$ & $-2,72$ \\
\hline 5 & 10116 & FEM1B & Fem-1 homolog b (C. elegans) & $-7,20$ & $-3,67$ & $-2,34$ \\
\hline 6 & 8772 & FADD & Fas (TNFRSF6)-associated via death domain & $-6,77$ & $-4,31$ & $-2,13$ \\
\hline 7 & 1050 & CEBPA & CCAAT/enhancer binding protein (C/EBP), alpha'"'" & $-6,23$ & $-4,57$ & $-2,19$ \\
\hline 8 & 10773 & ZNF482 & Zinc finger protein 482 & $-5,98$ & $-4,00$ & $-2,11$ \\
\hline 9 & 92342 & MGC9084 & Chromosome 1 open reading frame 156 & $-5,75$ & $-4,27$ & $-2,74$ \\
\hline 10 & 51058 & LOC51058 & Zinc finger protein 691 & $-5,61$ & $-4,22$ & $-2,01$ \\
\hline 11 & 51126 & NAT5 & N-acetyltransferase 5 (ARD1 homolog, S. cerevisiae)"'"' & $-5,35$ & $-5,73$ & $-2,31$ \\
\hline 12 & 5718 & PSMD12 & Proteasome (prosome, macropain) 265 subunit, non-ATPase, $12^{\prime \prime \prime \prime \prime}$ & $-4,91$ & $-3,51$ & $-1,95$ \\
\hline 13 & 7096 & TLR1 & Toll-like receptor 1 & $-4,81$ & $-4,18$ & $-2,53$ \\
\hline 14 & 26224 & FBXL3 & F-box and leucine-rich repeat protein 3 & $-4,67$ & $-4,09$ & $-3,01$ \\
\hline 15 & 57561 & ARRDC3 & Arrestin domain containing 3 & $-4,57$ & $-4,76$ & $-2,36$ \\
\hline 16 & 148479 & PHF13 & PHD finger protein 13 & $-4,51$ & $-3,72$ & $-2,59$ \\
\hline 17 & 10978 & HEAB & ATP/GTP-binding protein & $-4,51$ & $-3,24$ & $-2,59$ \\
\hline 18 & 7728 & ZNF175 & Zinc finger protein 175 & $-4,50$ & $-3,25$ & $-2,27$ \\
\hline 19 & 55330 & $\mathrm{CNO}$ & Cappuccino homolog (mouse) & $-4,49$ & $-4,60$ & $-1,96$ \\
\hline 20 & 79891 & FLJ23506 & Zinc finger protein 671 & $-4,43$ & $-3,17$ & $-2,39$ \\
\hline 21 & 57547 & ZNF624 & Zinc finger protein 624 & $-3,72$ & $-3,29$ & $-2,26$ \\
\hline 22 & 7568 & ZNF20 & Zinc finger protein 20 (KOX 13) & $-3,62$ & $-3,80$ & $-2,41$ \\
\hline 23 & 63915 & TXNDC5 & Thioredoxin domain containing 5 & $-3,57$ & $-2,66$ & $-2,42$ \\
\hline 24 & 57567 & ZNF319 & Zinc finger protein 319 & $-3,37$ & $-4,39$ & $-2,36$ \\
\hline 25 & 91574 & FLJ38663 & Hypothetical protein FLJ38663 & $-3,34$ & $-3,20$ & $-2,04$ \\
\hline 26 & 4064 & LY64 & CD180 antigen & $-3,11$ & $-3,23$ & $-3,00$ \\
\hline 27 & 874 & CBR3 & Carbonyl reductase 3 & $-3,03$ & $-4,09$ & $-2,01$ \\
\hline 28 & 9655 & SOCS5 & Suppressor of cytokine signaling 5 & $-2,85$ & $-2,29$ & $-2,33$ \\
\hline 29 & 10668 & CGRRF1 & Cell growth regulator with ring finger domain 1 & $-2,66$ & $-3,51$ & $-1,92$ \\
\hline 30 & 8799 & PEX11B & Peroxisomal biogenesis factor $11 \mathrm{~B}$ & $-2,64$ & $-2,52$ & $-2,44$ \\
\hline 31 & 132241 & WDR10 & Intraflagellar transport 122 homolog (Chlamydomonas) & $-2,62$ & $-2,52$ & $-2,67$ \\
\hline 32 & 901 & CCNG2 & Cyclin G2 & $-2,58$ & $-2,34$ & $-2,53$ \\
\hline
\end{tabular}

FDR 10. LM: L. monocytogenes EGDe, SA: S. aureus, SP: S. pneumoniae.

similarity between pneumococcal and LM-induced gene expression could be due to the cellular response to CDC-type toxins produced by these bacteria [12]. Nevertheless, there were clear differences in the number of detectable differentially regulated genes as well, with fewer genes being differentially expressed on infection with SP. This might point to an as yet unknown mechanism for subduing the host response by SP or it might indicate the improved immune evasion ability of this particular capsular SP strain. Remarkably, hallmark inflammatory cytokines, e.g. TNF and IL1 were not part of the common response of the monocytes. However, the most prominent feature of the common genes set is the upregulation of interleukin 23A (IL23, p19) mRNA. Thus it seems that in naive human monocytes grampositive bacteria induce the transcription of IL23 as the first major systemic proinflammatory cytokine, reminiscent of the effects of Mycobacteria and Salmonellae $[13,14]$. Real time RT-PCR experiments confirmed clearly the induction of IL23A (p19) mRNA as revealed by the microarray experiments. Moreover, the qRT-PCR also showed the upregulation of the IL23B (p40) gene, which however was very donor specific with a variation of 3 orders of magnitude (Figure 3; Additional file 16, Table S13). Thus infection of the monocytes with all gram-positive bacteria led to markedly increased 


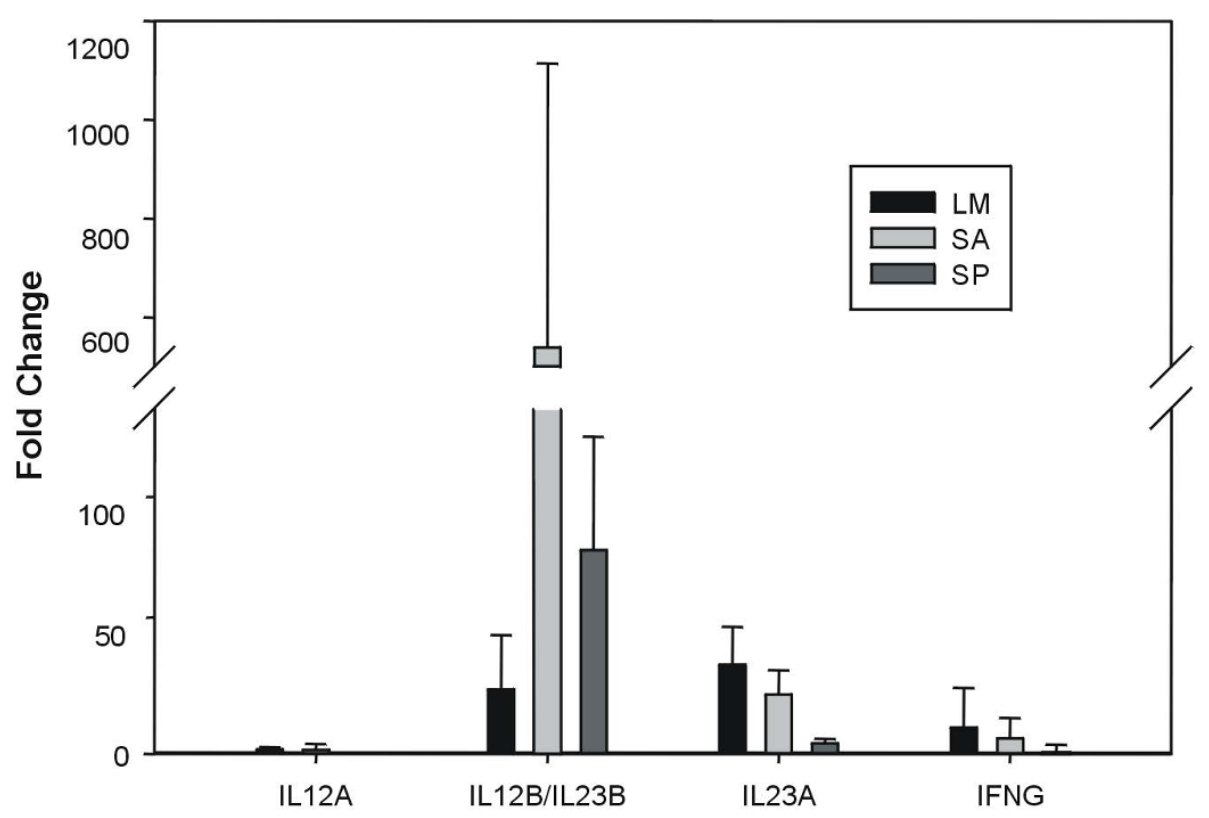

Figure 3 Relative quantification of IL12A, IL12B/IL23B, IL23A and IFN $\gamma$ by real time RT-PCR. Relative expression of IL12A, IL12B/IL23B, IL23A and IFN $\gamma$ (IFNG) mRNAs as determined by real time RT-PCR of infected vs. noninfected cells. Results are means plus standard deviation for all 5 donors. LM: L. monocytogenes EGDe, SA: S. aureus, SP: S. pneumoniae.

transcription of both genes necessary to form the active IL23 cytokine. At the same time microarray as well the qRT-PCR data showed only limited upregulation or even downregulation of the IL12A (p35) mRNA transcription for the individual donors, which confirms the dominant role of IL23 versus IL12. Both IL23 and IL12 can activate the transcription activator STAT4 in Thcells and NK cells, and stimulate the production of interferon $\gamma$ (IFN $\gamma$ ) [15,16]. However, as the monocyte population used in this study is almost free of other leukocytes including Th-cells and NK cells, the IFN $\gamma$ back loop that triggers secondary cytokine expression in the monocytes is absent, hence providing an explanation as to why other major inflammatory cytokines like IL1 and TNF were not substantially upregulated. Yet real time RT-PCR tests with IFN $\gamma$ specific primers revealed upregulation of IFN $\gamma$ mRNA by all three pathogens ( 9.5 fold upregulated by LM, 6.2 by SA and 1.8 fold by SP) suggesting an ability for self-priming of the monocytes even in the absence of additional effector cells.

The proinflammatory reaction with the hallmark upregulation of IL23 involved also the chemokines CCL8, CCL14 and osteopontin (3 to 9 fold upregulated and common for all pathogens). CCL14 weakly activates monocytes but induces the proliferation of CD34+ progenitor cells. CCL8 is chemotactic and is active on all leucocytes $[17,18]$. Osteopontin (SPP1, OPN) induces the migration of macrophages and dendritic cells to the site of inflammation [19]. This elevated transcription of chemokine genes is aided by the upregulation of downstream members of the inflammation signaling e.g. PDE4B, which is the predominant phosphodiesterase in macrophages and counteracts the inflammation inhibition by cyclic nucleotide signaling [20-22]. In circulating human monocytes PDE4B assists the TNFa synthesis and release in response to LPS $[21,22]$ thus our results show that upregulation of PDE4B can also be stimulated by alternative PAMP sensors since the gram positive bacteria we have used do not produce LPS.

Recently it was discovered that bacterial LPS and non methylated $\mathrm{CpG}$ oligonucleotides, which signal via TLR4 and TLR9 respectively, strongly induce the expression of SOCS1 and SOCS3, and attenuate the ability of macrophages to respond to subsequent stimulation by IFN $\gamma$ or IL-6 [23]. Similarly it has been described that NALP2 (PAN1) protein expression was also upregulated by LPS and interferons (IFN $\beta$ and IFN $\gamma$ ) and transient overexpression of NALP2 led to inhibition of NFkB signaling [24]. We also found that the upregulation of proinflammatory genes was accompanied by the upregulation of known anti-inflammatory proteins of the SOCS and NALP family (SOCS1, SOCS3, NALP2). This supports the concept of a dynamic equilibrium between inflammation induction and suppression in order to avoid excessive tissue damage. Clearly, gram-positive bacteria are also able to directly induce SOCS and NALP2 gene transcription but the actual pathway of signal transduction here must 
be attributed either only to TLR9 or another pathogenrecognition receptor, most likely TLR2 [25].

The microarray results also point to a novel and obviously important function of stimulated monocytes in angiogenesis and modulation of the peripheral vascular tonus. We observed the upregulation of transcription of the strong vasoactive mediators END1, VEGF and F3. Endothelin 1 (END1) is a potent vasoconstrictor and angiogenic peptide. Its expression has been attributed to damaged vascular endothelium, mast cells or macrophages in atherosclerotic lesions and thus it appears to be also a feature of stimulated monocytes in response to infection. The potential effect of endothelin induction also correlates with the upregulation of VEGF by all three pathogens. VEGF (vascular epithelium growth factor) is a major inducer of vascularization and angiogenesis $[26,27]$. In keeping with this observation we find that F3 (coagulation factor III thromboplastin tissue factor) is also overexpressed. Blood coagulation together with vasoconstriction ensures wound closing and prevents blood loss, but also prevents the invasion and spread of pathogens at the site of injury. Osteopontin (also upregulated) protects the endothelial cells against apoptosis and induces cell survival and proliferation. It also promotes migration of macrophages and dendritic cells to the site of inflammation and induces IL-12 secretion while down regulating the inducible nitric oxide synthase (iNOS) expression and the NO production by macrophages [19]. Our findings suggest that peripheral monocytes may have a very distinct role in processes of wound healing and the maintenance of environmental barriers when stimulated by bacterial pathogens. Interestingly some of the genes found upregulated in the monocytes were reported to have been regulated in endothelial cells upon treatment with VEGF: Egr3, Dusp4 [28] thus suggesting autocrine effects of VEGF (for LM and SA). Also the upregulation of VEGF in this study was two-fold for every single pathogen unlike the rest of upregulated cytokines and chemokines, which were usually more strongly upregulated by LM and SA. This may be interpreted as a sign for a very tight regulation of this growth factor, since another strong effect of VEGF is endothelium permeabilization, which may cause undesired exudate formation.

Another interesting characteristic of the common response was the upregulation of genes, known to counteract apoptotic signals and the absence of significant changes in the transcription of proapoptotic mediators. BIRC3 (upregulated by LM) inhibits apoptosis by binding to tumor necrosis factor receptor-associated factors TRAF1 and TRAF2 [29]. The protein encoded by TNFAIP3 is a zinc finger protein, and has been shown to inhibit TNF-mediated apoptosis [30]. Thioredoxin reductase 3 (TXNRD3) and superoxide dismutase 2
(SOD2) reduce effects of oxidative stress on mitochondria [31,32]. The upregulation of these genes indicates that the cells have to cope with higher radical production in the mitochondria caused by higher energy demands of the cell. On the other hand free radicals are produced by cytoplasmic stress and by lysosomal activity during infection. Accumulation of free radicals in the cytoplasm and in the mitochondria leads to activation of apoptotic pathways. Hence the upregulation of antiapoptotic genes and radical reducing enzymes as revealed above restores cell homeostasis and cell viability and suggests that at least at this early time point of infection the monocytes are actively suppressing an apoptotic program and are rather becoming primed for pathogen elimination and immune system activation.

The regulation of several genes was specifically influenced only by one of the pathogens. For example LM induced the transcription of FCAR (receptor for Fc fragment of $\operatorname{IgA}$ ), a member of the immunoglobulin gene superfamily. FCAR encodes a receptor for the Fc region of IgA present on the surface of myeloid lineage cells such as neutrophils, monocytes, macrophages, and eosinophils [33]. The activated receptor triggers several immunologic defense processes, including phagocytosis, antibody-dependent cell-mediated cytotoxicity, and release of inflammatory mediators. This finding suggests the ability of the monocytes to actively interact with IgA-opsonized pathogens, which is likely to happen at entry sites of bacteria at mucosal barriers, even when the monocytes have not become tissue resident yet.

SA specifically upregulated MC1R (melanocortin receptor 1). The expression of MC1R on monocytes was found to be upregulated by LPS and proinflammatory cytokines [34,35]. Activation of MC1R has been shown to cause a marked reduction of activation and translocation of the nuclear transcription factor NFkB, thus suggesting that $\alpha \mathrm{MSH}$ ( $\alpha$ melanocyte stimulating hormone) exerts its anti-inflammatory effect in part through activation of MC1R [36].

Surprisingly the overall transcriptional response to infection with SP was weaker compared to LM and SA, even though this strain is a clinical isolate from an infant with severe pneumococcal pneumonia. It appearss that SP relies on its ability to avoid or delay the full innate immune response, hence the smaller number and weaker upregulation of genes involved in the initiation of inflammation (IL23, CCL8, CCL14). Also the twofold induction of the immunosuppressive cytokine IL10 may contribute to the initial survival of the pathogen. On the other hand SP specifically induced two genes that are thought to have proinflammatory functions: CCL21 and CSF3. The protein encoded by the CCL21 gene is chemotactic in vitro for thymocytes and activated $\mathrm{T}$ cells, but not for $\mathrm{B}$ cells, macrophages, or 
neutrophils. The cytokine encoded by this gene may also play a role in mediating homing of lymphocytes to secondary lymphoid organs. CSF3 (granulocytes colony stimulation factor 3 ) is a cytokine that controls the production, differentiation, and function of granulocytes. We may speculate that the specific expression of the last two genes might contribute to severity of the inflammation at later stages of infection as caused by this pathogen in vivo.

\section{Conclusion}

We employed DNA expression microarrays to study the early transcriptional response of naive human peripheral monocytes infected with a set of three important grampositive bacterial pathogens: Staphylococcus aureus, Streptococcus pneumoniae and Listeria monocytogenes. Upregulation of chemokine rather than interleukin genes was characteristic for the early response with the exception of the prominent expression of IL23, marking it as the lead early cytokine. An important finding was the observed activation of genes regulating angiogenesis and endothelial cell function together with genes involved in managing pathogen induced cytoplasmic stress and counteracting apoptosis. This transcription program seems to be characteristic for the first events in monocyte activation and points to induction of cytokine signalling rather than to a program change of naïve monocytes to pathogen eliminating effector cells.

\section{Methods}

\section{Isolation of CD14 positive WBCs from human} peripheral blood

Blood concentrates (buffy coats) were obtained routinely at the transfusion center, clinic of JLU Gießen. Approval for the use of clinical material in this study was in compliance with procedures laid down by the Helsinki Declaration and approved by the Ethics Study Board of the University Hospital of Giessen (File number 79/01). For the isolation of monocytes, only fresh ( 1 to 1.5 hour old) buffy coats from phenotypic healthy donors (3 males +2 females) were used. The isolation of the mononuclear leucocytes was done by centrifugation trough a ficol cushion (Ficol-Plaque-TM, Amersham Biosciences). After the centrifugation the interphase was collected and the cells were washed twice with PBS. The cells were reconstituted in PBS and kept on ice. AntiCD14 antibody labeled magnetic beads (Miltenyi Biotec, Bergisch Gladbach, Germany) were added to the cells in a ratio of $20 \mu \mathrm{l} / 10^{7}$ cells (ca. $5 \mathrm{Abs} . / \mathrm{cell}$ ). After $15 \mathrm{~min}$. incubation at $4{ }^{\circ} \mathrm{C}$ unbound beads were separated by a short centrifugation step and the labeled cells were loaded and purified on a LS positive selection column using the MidiMACS magnetic separator (Miltenyi Biotec, Bergisch Gladbach, Germany) following the manufacturers instruction. The CD14+ cells were eluted in PBS and an aliquot was used for cell counting. The cells suspension was filled up with room temperetured RPMI medium (1\% FCS) and portions of $5 \times 10^{6}$ cells/ well were seated into a 6 -well tissue culture dish. The cells were allowed to adhere to the plate bottom for 45 min at $37^{\circ} \mathrm{C}$ in a $\mathrm{CO}_{2}$ tissue culture incubator.

\section{FACS analysis of isolated cells}

Monoclonal FITC-labeled Antibodies were ordered from Miltenyi Biotec: anti CD14 clone TÜK4 and Immunotools (Friesoythe; Germany): anti CD11b-clone MEM174. $1 \mu \mathrm{l}$ anti CD14-FITC and $3 \mu \mathrm{l}$ anti CD11b-FITC antibody were diluted in $50 \mu \mathrm{l}$ of PBS, containing $0,5 \%$ BSA. $1 \times 10 \mathrm{e} 6$ cells were added to each diluted antibody and were incubated for $30 \mathrm{~min}$. at $4^{\circ} \mathrm{C}$. After the incubation the cells were washed three times with $2 \mathrm{ml}$ PBS/ BSA by centrifugation for $5 \mathrm{~min}$. at $400 \mathrm{~g}$. Afterwards the cells were recovered in $0.5 \mathrm{ml}$ of PBS/BSA and measured on a FACScalibure flow cytometer (BD, Heidelberg, Germany). The flow cytometer measurement revealed $12 \%$ CD14 and 28\% CD11b positive cells in the mononuclear cell fraction after ficol gradient separation. The magnetic beads purified cells were enriched to $96 \%$ CD14+ and 98\% CD11b+ respectively. Thus the magnetic bead separation produced a highly enriched monocyte fraction (Additional file 17, Figure S2).

\section{Bacterial cultures and infection assay}

L. monocytogenes EGDe is a serotype 1/2a wild type isolate as described by Glaser P et al. 2001 [37]. S. aureus Gi.11268 and S. pneumoniae Gi.15342 are patient isolates characterized at the Institute of Medical Microbiology, Giessen. Overnight culture of L. monocytogenes EGDe and S. aureus Gi.11268 were grown in BHI medium at $37^{\circ} \mathrm{C}$ by continuous shaking. The over night cultures were diluted 1:50 and bacteria were grown in $\mathrm{BHI}$ medium reaching an $\mathrm{OD}_{600}$ of 0.4 to 0.7 . The number of viable bacteria was calculated using growth curves for both organisms. S. pneumoniae Gi.15342 was prepared by washing the bacteria with prewarmed PBS from the surface of a Columbia-agar plate with an over night Streptococcus culture. The number of viable bacteria was calculated by using a dilutions curve at $\mathrm{OD}_{600}$. The required bacteria were collected by centrifugation at $5000 \mathrm{~g}$ for $10 \mathrm{~min}$. and reconstituted in RPMI medium containing $1 \%$ FCS to a final concentration of $5 \times 10^{7}$ bacteria $/ 100 \mu \mathrm{l}$. Adherent CD14+ cells were infected by adding $100 \mu \mathrm{l}$ of the diluted bacteria suspension yielding a moi of 10 . The tissue culture plaques were swung gently to mix the infectious medium and than centrifuged for $1 \mathrm{~min}$ at $900 \mathrm{~g}$ to ensure an even contact of the bacteria with the cells. 2 to 3 control wells received 
$100 \mu \mathrm{l}$ of sterile medium. The cells were incubated for $1 \mathrm{~h}$ in a $\mathrm{CO}_{2}$ tissue culture incubator followed by cell lysis and RNA isolation. No antibiotics were used by the preparation of the cells and during the infection.

\section{RNA isolation}

For every bacterial pathogen and negative control the cells of at least two wells of a six well tissue culture plaque were lysed and total RNA was isolated. Prior to lysis culture medium was aspirated and cells lysed using RLT lysis buffer (Qiagen, Hilden, Germany). Total RNA was isolated using the RNeasy Mini Kit and the RNase free DNase I set (Qiagen) following the manufacturers protocol. The RNA was recovered in RNase free water, heat denatured for $10 \mathrm{~min}$. at $65^{\circ} \mathrm{C}$; quantified with the NanoDrop ${ }^{\circ}$ ND-1000 UV-Vis Spectrophotometer (NanoDrop Technologies, Rockland DE, USA) and a quality profile with the Agilent 2100 bioanalyzer (Agilent Technologies GmbH, Waldbronn, Germany) was made.

\section{CodeLink target labeling and array hybridization}

Target preparation was done using the "CodeLink Expression Assay Reagent Kit" Manual Prep (Amersham Biosciences, Chandler AZ, USA) and the original protocol for CodeLink System manual target preparation (Amersham Biosciences, Chandler AZ, USA). Briefly: $2 \mu \mathrm{g}$ total RNA were used in cDNA synthesis reaction with a poly-A binding primer containing the T7polymerase promoter. Clean up of the resulting dsDNA fragments was done using the QIAquick PCR Purification Kit (Qiagen, Hilden, Germany). For target labeling the cDNA was in vitro transcribed by partially substituting UTP with bio-16-UTP in the reaction mixture. Labeled cRNA was purified using the RNeasy Mini Kit (Qiagen, Hilden, Germany). Portions of $20 \mu \mathrm{g}$ cRNA were subjected to fragmentation in the presence of $\mathrm{Mg}^{2+}$. Subsequently $10 \mu \mathrm{g}$ fragmented cRNA (target) was loaded onto UniSet Human I BioArray glass slides ( $\mathrm{n}=2$ arrays per sample) and hybridized for $18 \mathrm{~h}$ in a Minitron shaker incubator (Infors AG, Bottmingen, Germany) at $37 \mathrm{C}^{\circ} / 300 \mathrm{rpm}$. Washing and dyeing with $\mathrm{Cy}-5$ coupled streptavidin (Amersham Biosciences, Freiburg, Germany) was done according to the original protocol and the arrays were scanned using an GenePix 4000 B scanner and GenePix Pro 4.0 Software (Axon Instruments, Arlington, USA).

\section{Microarray data analysis}

Images were analyzed using CodeLink Expression Analysis Software. Data was normalized by quantile normalization [38]. Data was $\log 2$ transformed and spots that were always flagged EMPTY were removed. Spots that were flagged empty across all technical replicates were discarded. All spots except the DISCOVERY spots were also discarded. The missing values were imputed using SeqKNN [39]. Technical replicates were averaged. Differentially expressed genes were detected using Rank Products [40], both at False Discovery Rate 5 and 10, as an unpaired analysis for each treatment being compared to the untreated control chips. The resulting gene list was subjected to DAVID and EASE [41] for annotation and overrepresentation analysis of gene categories. Due to the highly similar expression profiles of all donors to every single pathogen the microarray results presented in all tables are the mean fold change for the donor pool. The microarray data has been submitted to the ArrayExpress database and can be accessed using the accession number E-MEXP-1613.

\section{Real time RT-PCR}

First-strand cDNA was synthesized with 500 ng of purified RNA using SuperScriptII (Invitrogen) and a mixture of T21 and random nonamer primers (Metabion) following the instructions for the reverse transcription reaction recommended for the QuantiTect SYBR Green Kit (Qiagen). Real-time quantitative PCR was performed with QuantiTect SYBR Green Kit (Qiagen) on an ABI Prism 7700 real time cycler. The relative expression of 14 target genes was normalized to that of a pool of four reference genes. PCR primers were either self-validated or commercially available QuantiTect primer assays (Qiagen). Primer sequence for the self-validated primers was as follows B2M-forward: 5'-TCTTTTTCAGTGGGGGTGA-3', B2M-reverse: 5'-TCCATCCGACATTGAAGTT-3', G6PD-forward: 5' - AGCAGTGGGGTGAAAATAC-3', G6PD-reverse: 5'-CCTGACCTACGGCAACAGA-3', TLR1-forward: 5'-TAATTTTGGATGGGCAAAGC-3', TLR1-reverse: 5'-CACCAAGTTGTCAGCGATGT-3'. For every target and reference gene a standard dilution curve with a reference RNA sample was done and the linear equation was used to transform threshold cycle values into nanograms of total RNA [42]. The relative fold change of target genes in the infected samples versus the non-treated control was normalized by the relative expression of a pool of 4 reference genes: B2M (Beta 2 microglobulin), G6PD (Glucose 6 phosphate dehydrogenase), PGK1 (Phosphoglycerate kinase 1) and SDHA (Succinate dehydrogenase alpha subunit). Normalized fold change for a target gene versus every reference gene was calculated and a mean fold change of these four was the final value.

\section{Additional material}

Additional file 1: Table S1. L. monocytogenes - Totally upregulated genes. FDR 10. 
Additional file 2: Table S2. L. monocytogenes - Totally downregulated genes. FDR 10

Additional file 3: Table S3. S. aureus - Totally upregulated genes. FDR

Additional file 4: Table S4. S. aureus - Totally downregulated genes. FDR 10

Additional file 5: Table S5. S. pneumoniae - Totally upregulated genes. FDR 10

Additional file 6: Table S6. S. pneumoniae - Totally downregulated genes. FDR 10

Additional file 7: Table S7. L. monocytogenes - Specifically upregulated genes. FDR 10

Additional file 8: Table S8. L. monocytogenes - Specifically

downregulated genes. FDR 10

Additional file 9: Table S9. S. aureus - Specifically upregulated genes. FDR 10

Additional file 10: Table S10. S. aureus - Specifically downregulated genes. FDR 10

Additional file 11: Table S11. S. pneumoniae - Specifically upregulated genes. FDR 10

Additional file 12: Table S12. S. pneumoniae - Specifically

downregulated genes. FDR 10

Additional file 13: Excel work sheet S1. Differentially expressed genes by all three pathogens with $\mathrm{GO}$ grouping.

Additional file 14: Excel work sheet S2. Most and least variable genes in the none challenged cells classified by Gene Ontology.

Additional file 15: Figure S1. Correlation of Fold Change. Relative expression of 14 genes as determined by real time RT-PCR upon infection plotted against their corresponding microarray values. Results are averaged for all 5 donors

Additional file 16: Table S13. Relative gene expression of IL12A, IL12B/ IL23B, IL23A and IFN $\gamma$, detected by real time RT-PCR

Additional file 17: Figure S2. Phenotype of peripheral mononuclear cells before and after CD14+ positive selection. Anti CD11b and anti CD14 antibodies labeling after ficol gradient centrifugation and before and after CD14 positive selection. Percent of positive cells from all viable mononuclear cells. (A) CD11b $+: 28 \%$ before and $98 \%$ positive cells after CD14 + selection. (B) CD14+ : 12\% before and 96\% positive cells after CD14 + selection

\section{Acknowledgements}

The authors wish to thank Juri Schklarenko for excellent technical assistance, Prof. Dr. Gregor Bein (Institute of Clinical Immunology and Transfusion Medicine, University Clinic of Giessen) for providing the buffycoats and Andre Billion (Institute of Medical Microbiology, University of Giessen) for helping editing the figures.

The study was funded by grants from the National Genome Research Network (NGFN) through the Bundesministerium für Bildung und Forschung (BMBF) to T.C.

\section{Author details}

1 Institute of Medical Microbiology, Justus Liebig University, Frankfurter Str. 107, 35392 Giessen, Germany. ${ }^{2}$ Evolutionary Genomics Group, Departamento Producción Vegetal y Microbiología, Universidad Miguel Hernández, San Juan de Alicante, Spain.

\section{Authors' contributions}

ST performed the experimental work and wrote the manuscript. RG participated in the statistical analysis of microarray data and in writing the manuscript. $\mathrm{HH}$ participated in the statistical analysis of microarray data and in writing the manuscript. TC conceived the study and helped drafting the manuscript. All authors have read and approved the final manuscript.
Received: 4 May 2010 Accepted: 2 November 2010

Published: 2 November 2010

\section{References}

1. Bone RC: Gram-positive organisms and sepsis. Arch Intern Med 1994, 154:26-34.

2. Cohen J, Abraham E: Microbiologic findings and correlations with serum tumor necrosis factor-alpha in patients with severe sepsis and septic shock. J Infect Dis 1999, 180:116-121.

3. Luzzaro F, Viganò EF, Fossati D, Grossi A, Sala A, Sturla C, Saudelli M, Toniolo A: Prevalence and drug susceptibility of pathogens causing bloodstream infections in northern Italy: a two-year study in 16 hospitals. Eur J Clin Microbiol Infect Dis 2002, 21:849-55.

4. Nicoletti G, Schito G, Fadda G, Boros S, Nicolosi D, Marchese A, Spanu T, Pantosti A, Monaco M, Rezza G, Cassone A, Garaci E: Bacterial isolates from severe infections and their antibiotic susceptibility patterns in Italy: a nationwide study in the hospital setting. J Chemother 2006, 8:589-602.

5. Bindayna KM, Jamsheer A, Farid E, Botta GA: Neonatal sepsis 1991-2001: prevalent bacterial agents and antimicrobial susceptibilities in Bahrain. Med Princ Pract 2006, 15:131-6.

6. Draper DW, Bethea HN, He YW: Toll-like receptor 2-dependent and -independent activation of macrophages by group $\mathrm{B}$ streptococci. Immunol Lett 2006, 102:202-214.

7. Feezor RJ, Oberholzer C, Baker HV, Novick D, Rubinstein M, Moldawer LL, Pribble J, Souza S, Dinarello CA, Ertel W, Oberholzer A: Molecular characterization of the acute inflammatory response to infections with gram-negative versus gram-positive bacteria. Infect Immun 2003, 71:5803-5813.

8. Moreilhon C, Gras D, Hologne C, Bajolet O, Cottrez F, Magnone, Merten M, Groux H, Puchelle E, Barbry P: Live Staphylococcus aureus and bacterial soluble factors induce different transcriptional responses in human airway cells. Physiol Genomics 2005, 20:244-255.

9. Rogers PD, Thornton J, Barker KS, McDaniel DO, Sacks GS, Swiatlo E, McDaniel LS: Pneumolysin-dependent and -independent gene expression identified by CDNA microarray analysis of THP-1 human mononuclear cells stimulated by Streptococcus pneumoniae. Infect Immun 2003, 71:2087-2094.

10. Wang JE, Jorgensen PF, Almlof M, Thiemermann C, Foster SJ, Aasen AO, Solberg R: Peptidoglycan and lipoteichoic acid from Staphylococcus aureus induce tumor necrosis factor alpha, interleukin 6 (IL-6), and IL-10 production in both $\mathrm{T}$ cells and monocytes in a human whole blood model. Infect Immun 2000, 68:3965-3970.

11. Jenner $R G$, Young RA: Insights into host responses against pathogens from transcriptional profiling. Nat Rev Microbiol 2005, 3:281-294.

12. Winn W Jr, Allen S, Janda W, Koneman E, Procop G, Schreckenberger P, Woods G: Koneman's Atlas and Textbook of diagnostic microbiology 6-th edt. Lippincott Williams \& Wilkins; 2006, 631-637, 689-690, 766768.

13. Verreck FA, de Boer $T$, Langenberg DM, Hoeve MA, Kramer M, Vaisberg $E$, Kastelein R, Kolk A, de Waal-Malefyt R, Ottenhoff TH: Human IL-23producing type 1 macrophages promote but IL-10-producing type 2 macrophages subvert immunity to (myco)bacteria. Proc Natl Acad Sci USA 2004, 101:4560-4565.

14. Ottenhoff TH, Verreck FA, Lichtenauer-Kaligis EG, Hoeve MA, Sanal O, van Dissel JT: Genetics, cytokines and human infectious disease: lessons from weakly pathogenic mycobacteria and salmonellae. Nat Genet 2002, 32:97-105.

15. Mosser DM: The many faces of macrophage activation. J Leukocyte Biol 2003, 73:209-212.

16. Gordon S: Alternative activation of macrophages. Nat Rev Immunol 2003 3:23-35

17. Coelho AL, Hogaboam CM, Kunkel SL: Chemokines provide the sustained inflammatory bridge between innate and acquired immunity. Cytokine Growth Factor Rev 2005, 16:553-560.

18. Laing KJ, Secombes CJ: Chemokines. Dev Comp Immunol 2004, 28:443-460.

19. Chakraborty G, Jain S, Behera R, Ahmed M, Sharma P, Kumar V, Kundu GC: The multifaceted roles of osteopontin in cell signaling, tumor progression and angiogenesis. Curr Mol Med 2006, 6:819-830.

20. Erdely A, Kepka-Lenhart D, Clark M, Zeidler-Erdely P, Poljakovic M Calhoun WJ, Morris SM Jr: Inhibition of phosphodiesterase 4 amplifies 
cytokine-dependent induction of arginase in macrophages. Am J Physiol Lung Cell Mol Physiol 2006, 290:L534-L539.

21. Jin SL, Conti M: Induction of the cyclic nucleotide phosphodiesterase PDE4B is essential for LPS-activated TNF-alpha responses. Proc Natl Acad Sci USA 2002, 99:7628-7633.

22. Jin SL, Lan L, Zoudilova M, Conti M: Specific role of phosphodiesterase $4 B$ in lipopolysaccharide-induced signaling in mouse macrophages. J Immunol 2005, 175:1523-1531.

23. Ilangumaran S, Ramanathan S, Rottapel R: Regulation of the immune system by SOCS family adaptor proteins. Semin Immunol 2004, 16:351-365.

24. Bruey JM, Bruey-Sedano N, Newman R, Chandler S, Stehlik C, Reed JC: PAN1/NALP2/PYPAF2, an inducible inflammatory mediator that regulates NF-kappaB and caspase-1 activation in macrophages. J Biol Chem 2004, 279:51897-51907.

25. Machata S, Tchatalbachev S, Mohamed W, Jänsch L, Hain T, Chakraborty T: Lipoproteins of Listeria monocytogenes are critical for virulence and TLR2-mediated immune activation. J Immunol 2008, 181:2028-35.

26. Byrne AM, Bouchier-Hayes DJ, Harmey JH: Angiogenic and cell survival functions of vascular endothelial growth factor (VEGF). J Cell Mol Med 2005, 9:777-794

27. Takahashi H, Shibuya M: The vascular endothelial growth factor (VEGF)/ VEGF receptor system and its role under physiological and pathological conditions. Clin Sci (Lond) 2005, 109:227-241.

28. Liu D, Jia H, Holmes DI, Stannard A, Zachary I: Vascular endothelial growth factor-regulated gene expression in endothelial cells: KDR-mediated induction of Egr3 and the related nuclear receptors Nur77, Nurr1, and Nor1. Arterioscler Thromb Vasc Biol 2003, 23:2002-2007.

29. Uren AG, Pakusch M, Hawkins CJ, Puls KL, Vaux DL: Cloning and expression of apoptosis inhibitory protein homologs that function to inhibit apoptosis and/or bind tumor necrosis factor receptor-associated factors. Proc Natl Acad Sci USA 1996, 93:4974-4978.

30. Song HY, Rothe M, Goeddel DV: The tumor necrosis factor-inducible zinc finger protein A20 interacts with TRAF1/TRAF2 and inhibits NF-kappaB activation. Proc Natl Acad Sci USA 1996, 93:6721-6725.

31. Sun QA, Kirnarsky L, Sherman S, Gladyshev VN: Selenoprotein oxidoreductase with specificity for thioredoxin and glutathione systems. Proc Natl Acad Sci USA 2001, 98:3673-3678.

32. Miao L, St Clair DK: Regulation of superoxide dismutase genes: implications in disease. Free Radic Biol Med 2009, 47:344-356.

33. Morton HC, Brandtzaeg P: CD89: the human myeloid IgA Fc receptor. Arch Immunol Ther Exp 2001, 49:217-29.

34. Luger TA, Scholzen T, Brzoska T, Becher E, Slominski A, Paus R: Cutaneous immunomodulation and coordination of skin stress responses by alphamelanocyte-stimulating hormone. Ann N Y Acad Sci 1998, 840:381-394.

35. Luger TA, Scholzen TE, Brzoska T, Bohm M: New insights into the functions of alpha-MSH and related peptides in the immune system. Ann N Y Acad Sci 2003, 994:133-140.

36. Maaser C, Kannengiesser K, Specht C, Lugering A, Brzoska T, Luger TA, Domschke W, Kucharzik T: Crucial role of the melanocortin receptor MC1R in experimental colitis. Gut 2006, 55:1415-1422.

37. Glaser P, Frangeul L, Buchrieser C, Rusniok C, Amend A, Baquero F, Berche $P$, Bloecker H, Brandt $P$, Chakraborty $T$, Charbit A, Chetouani F, Couvé $E$, de Daruvar A, Dehoux P, Domann E, Domínguez-Bernal G, Duchaud E, Durant L, Dussurget O, Entian KD, Fsihi H, García-del Portillo F, Garrido P, Gautier L, Goebel W, Gómez-López N, Hain T, Hauf J, Jackson D, Jones LM, Kaerst $U$, Kreft J, Kuhn M, Kunst F, Kurapkat G, Madueno E, Maitournam A, Vicente JM, Ng E, Nedjari H, Nordsiek G, Novella S, de Pablos B, Pérez-Diaz JC, Purcell R, Remmel B, Rose M, Schlueter T, Simoes N, Tierrez A, Vázquez-Boland JA, Voss H, Wehland J, Cossart P: Comparative genomics of Listeria species. Science 2001, 294:849-852.

38. Bolstad BM, Irizarry RA, Astrand M, Speed TP: A comparison of normalization methods for high density oligonucleotide array data based on variance and bias. Bioinformatics 2003, 19:185-193.

39. Kim KY, Kim BJ, Yi GS: Reuse of imputed data in microarray analysis increases imputation efficiency. BMC Bioinformatics 2004, 5:160.

40. Breitling $R$, Armengaud P, Amtmann A, Herzyk P: Rank products: a simple, yet powerful, new method to detect differentially regulated genes in replicated microarray experiments. FEBS Lett 2004, 573:83-92.
41. Dennis G Jr, Sherman BT, Hosack DA, Yang J, Gao W, Lane HC, Lempicki RA: DAVID: Database for Annotation, Visualization, and Integrated Discovery. Genome Biol 2003, 4:3.

42. Critical factors for successful Real time PCR. Qiagen; 2004.

doi:10.1186/1471-2180-10-275

Cite this article as: Tchatalbachev et al:: Gram-positive pathogenic bacteria induce a common early response in human monocytes. BMC Microbiology 2010 10:275

\section{Submit your next manuscript to BioMed Central and take full advantage of:}

- Convenient online submission

- Thorough peer review

- No space constraints or color figure charges

- Immediate publication on acceptance

- Inclusion in PubMed, CAS, Scopus and Google Scholar

- Research which is freely available for redistribution

Submit your manuscript at www.biomedcentral.com/submit 